\title{
Involvement of DR4/JNK pathway-mediated autophagy in acquired TRAIL resistance in HepG2 cells
}

\author{
SUNG-CHUL LIM ${ }^{1}$, HO JONG JEON ${ }^{1}$, KEUN HONG KEE ${ }^{1}$, MI JA LEE ${ }^{1}$, RAN HONG $^{1}$ and SONG IY HAN ${ }^{2}$ \\ ${ }^{1}$ Department of Pathology, ${ }^{2}$ Division of Premedical Science, College of Medicine, \\ Chosun University, Gwangju 61501, Republic of Korea
}

Received July 7, 2016; Accepted September 8, 2016

DOI: $10.3892 /$ ijo.2016.3699

\begin{abstract}
Although tumor necrosis factor-related apoptosisinducing ligand (TRAIL) is a promising anticancer agent, a number of cancer cells demonstrate TRAIL resistance. To date, various molecular targets leading to TRAIL resistance have been elucidated by many researchers, but the mechanisms involved are still not fully understood. In the present study, we obtained TRAIL-resistant cells from the human hepatocellular carcinoma cell line HepG2 by exposing cells to recombinant human TRAIL (rhTRAIL), and determined a mechanism for TRAIL resistance. The selected TRAILresistant cells (HepG2-TR) were insensitive to rhTRAIL and triggered autophagy in response to rhTRAIL. The inhibition of autophagy by 3-methyladenine or the knockdown of ATG5 partially restored rhTRAIL-induced apoptosis and cytotoxicity, indicating that protective autophagy occurred in the cells. Notably, rhTRAIL-induced autophagy was mediated through DR4 in HepG2-TR cells, but not in parental HepG2 cells. In addition, the c-Jun $\mathrm{N}$-terminal kinase was involved in DR4-mediated autophagy in HepG2-TR cells. Our results suggest a novel mechanism of TRAIL resistance which is regulated through alterations in DR4 function, which may extend our understanding of the mechanisms of TRAIL resistance.
\end{abstract}

\section{Introduction}

The tumor necrosis factor-related apoptosis-inducing ligand (TRAIL) is an attractive anticancer agent, since it selectively induces apoptosis in a variety of cancer cells (1-4). Furthermore, TRAIL exhibits potent tumoricidal activity against tumor xenografts, without serious side-effects in preclinical studies $(2,3)$, implicating the potential utility of TRAIL in the treatment of human cancer.

TRAIL-induced apoptosis is initiated by the binding of TRAIL to its functional death receptors, TRAIL-R1 (DR4)

Correspondence to: Professor Song Iy Han, Division of Premedical Science, College of Medicine, Chosun University, Gwangju 61501, Republic of Korea

E-mail: sihan@chosun.ac.kr

Key words: TRAIL, resistance, autophagy, apoptosis and TRAIL-R2 (DR5). These TRAIL receptors have death domains (DD) in their cytoplasmic tails, which recruit the adaptor protein Fas-associated death domains (FADD) and trigger the formation of the death-inducing signaling complex (DISC). In the DISC, the recruited pro-forms of initiator caspases, such as caspase-8, are activated and subsequently trigger the activation of downstream effector caspases with or without mitochondrial amplification via cleavage of the BH3-only Bcl-2 family member Bid. TRAIL can also bind the decoy receptors TRAIL-R3 (DcR1) and TRAIL-R4 (DcR2), which possess incomplete cytoplasmic regions, and the soluble receptor osteoprotegerin. These receptors may inhibit the apoptotic pathway by competing for TRAIL with active death receptors (5). Higher expression of decoy receptors in nontransformed cells is considered one of the mechanisms that confer TRAIL selectivity to cancer cells. In addition, multiple pathways, including cellular FLICE-like inhibitory protein (c-FLIP), anti-apoptotic B-cell lymphoma 2 proteins (Bcl-2), and X-linked inhibitor of apoptosis protein (XIAP) pathways, may confer insensitivity to TRAIL in non-transformed cells (6). However, these mechanisms can also be utilized in resistant cancer cells to counteract to the antitumor effect of TRAIL. In fact, decoy R, c-FLIP and Bcl-2 are overexpressed in several TRAIL-resistant cancer cells (7-9). In addition, resistance to TRAIL could be gained at various other steps in the signaling pathways of apoptosis.

Currently, TRAIL resistance in cancer cells has emerged as the major obstacle to successful TRAIL therapy. To date, the mechanisms underlying TRAIL resistance have been mostly studied in TRAIL-sensitive cancer cells to understand how sensitive cells acquire TRAIL resistance. However, it is also important to understand how less sensitive cancer cells can gain much stronger resistance to TRAIL after TRAIL exposure, since cancers frequently display heterogeneous features, and even cancer cells with primary resistance could develop stronger resistance via various mechanisms after exposure to TRAIL. In this study, we observed an unexpected role of DR4 in TRAIL-resistant HepG2 cells and examined the mechanism underlying DR4-mediated resistance in HepG2-TR cells.

\section{Materials and methods}

Cell culture. The HepG2 HCC cell line, AGS and SNU601 gastric cancer cell lines, and HCT116 colon cancer cell line 
were obtained from the Korean Cell Line Bank (Seoul, Korea) and cultured in RPMI-1640 medium (Invitrogen, Carlsbad, CA, USA) supplemented with $10 \%$ (v/v) fetal bovine serum (FBS) and $1 \%$ antibiotics at $37^{\circ} \mathrm{C}$ in a $5 \% \mathrm{CO}_{2}$ atmosphere. A TRAIL-resistant variant of HepG2 cells (HepG2-TR) was obtained by periodically exposing the HepG2 cell line to increasing concentrations $(5-100 \mathrm{ng} / \mathrm{ml})$ of recombinant human TRAIL (rhTRAIL; a gift from T.H. Kim, Department of Biochemistry and Molecular Biology, Chosun University, Gwangju, Korea) and selecting for resistance to TRAILinduced apoptosis.

Cell viability assays. For cell viability detection, cells were plated in a 96-well plate at a density of $1 \times 10^{4}$ cells/well, incubated for $24 \mathrm{~h}$, and then treated with rhTRAIL for $48 \mathrm{~h}$. Next, 3-(4,5-dimethylthiazol-2-yl)-2,5-diphenyltetrazolium bromide (MTT) solution $(0.5 \mathrm{mg} / \mathrm{ml})$ was added to the wells and plates were incubated at $37^{\circ} \mathrm{C}$ in a $\mathrm{CO}_{2}$ incubator for $4 \mathrm{~h}$. The plates were centrifuged at $600 \mathrm{x}$ g for $10 \mathrm{~min}$ and the culture medium was removed. The cells were solubilized using dimethyl sulfoxide and the solubilized formazan product was quantified using an enzyme-linked immunosorbent assay plate reader at $595 \mathrm{~nm}$. The absorbance of the untreated cells was set to $100 \%$ and cell survival was expressed as a percentage of this value.

Apoptosis analysis. For Hoechst 33342 staining, cells were stained with $1 \mu \mathrm{g} / \mathrm{ml}$ Hoechst 33342 for $15 \mathrm{~min}$ at room temperature in the dark, and both the floating and attached cells were collected and centrifuged. The pooled cell pellets were washed with ice-cold phosphate-buffered saline (PBS), fixed in $3.7 \%$ formaldehyde on ice, washed and resuspended in PBS, and then a fraction of the suspension was centrifuged using a cyto-spinner (Shandon; Thermo Fisher Scientific, Waltham, MA, USA). Slides were prepared, air dried, mounted in anti-fade solution, and observed under a fluorescence microscope (DM5000; Leica Microsystems, Washington DC, USA), as described elsewhere. Any condensed/fragmented nuclei were assessed as apoptotic cells. In total, 500 cells distributed across random microscope viewing fields were counted, and the number of apoptotic cells is expressed as a percentage of the total number of cells counted. For the flow cytometric analysis, cells were washed with $1 \%$ Triton X-100/PBS and fixed with cold methanol for $30 \mathrm{~min}$. After washing, fixed cells were incubated with $20 \mu \mathrm{g} / \mathrm{ml}$ DNAse-free RNase A for $30 \mathrm{~min}$ at $37^{\circ} \mathrm{C}$, then with $50 \mu \mathrm{g} / \mathrm{ml}$ propidium iodide for an additional $40 \mathrm{~min}$ on ice. The cells were washed and subjected to fluorescence-activated cell sorting analysis (NAVIOS flow cytometer; Beckman Coulter, Inc., Brea, CA, USA).

Autophagy analysis. For autophagy detection, the CYTO-ID Autophagy detection kit was used according to the manufacturer's instruction (Enzo Life Sciences, Farmingdale, NY, USA). In brief, cells cultured on a 96-well microplate were treated with TRAIL and a positive control (a combination of rapamycin and chloroquine) for $48 \mathrm{~h}$. After treatment, the medium was removed and cells were rinsed with assay buffer. Then, cells were incubated with dual-color detection solution (CYTO-ID Green/Hoechst 33342) diluted in assay buffer at $37^{\circ} \mathrm{C}$ for $30 \mathrm{~min}$, rinsed twice, and analyzed with a fluorescence microplate reader. CYTO-ID Green was detected using a FITC filter $(\mathrm{Ex}=488 / \mathrm{Em}=530)$ and Hoechst 33342 was read with a DAPI filter set $(E x=340 / E m=480)$. The relative green fluorescence intensity was normalized against nuclear blue fluorescence intensity. To observe autophagic vacuoles by fluorescence microscopy, treated cells were incubated with $10 \mu \mathrm{M}$ monodansylcadaverine for $30 \mathrm{~min}$, washed with PBS, and observed under an inverted fluorescence microscope at $340 / 525 \mathrm{~nm}$.

Immunoblotting. Equal amounts of protein extracts were electrophoretically separated using 10-12\% SDS-PAGE and transferred to a nitrocellulose membrane using standard techniques. Antibodies were used to probe for DR4, DR5 (ProSci, Inc., Poway, CA, USA), caspase-3, caspase-8, phospho-JNK1/2, total JNK1/2 (Cell Signaling Technology, Danvers, MA, USA), cytochrome $c$, caveolin-1 (Santa Cruz Biotechnology, Santa Cruz, CA, USA), and LC3 (Novus Biologicals, Littleton, CO, USA). Anti- $\alpha$-tubulin (BioGenex, Fremont, CA, USA) was used as a loading control. Signals were acquired using an Image Station 4000MM Image Analyzer (Kodak, Rochester, NY, USA).

RNA interference (RNAi). For the RNAi experiment, siRNA targeting DR4 (\#1), 5'-CUGGAAAGUUCAUCUACUU (dtdt)-3' (sense) and 5'-AAGUAGAUGAACUUUCCAG (dtdt)-3' (antisense); DR5, 5'-CAGACUUGGUGCCCUUUG (dtdt)-3' (sense) and 5'-UCAAAGGGCACCAAGUCUG (dtdt)-3' (antisense), and 5'-control siRNA, 5'-CCUACGCCAC CAAUUUCGU(dtdt)-3' (sense) and 5'-ACGAAAUUGGUG GCGUAGG (dtdt)-3' (antisense) were purchased from Bioneer (Daejeon, Korea). siRNA targeting DR4 (\#2) (sc-35218) was purchased from Santa Cruz Biotechnology. Cells were individually transfected with siRNA oligonucleotides using the jetPEI transfection reagent (Polyplus-transfection SA, Illkirch, France) and grown for $36 \mathrm{~h}$ prior to the drug treatment.

Statistical analysis. All numerical data are reported as means \pm SE. All data represent the results of at least three independent experiments. Student's t-tests were used to evaluate the differences in means between control and treatment groups, and one-way ANOVA was applied to analyze differences caused by gene silencing or inhibitor treatment.

\section{Results}

Exposure to rhTRAIL increases the TRAIL resistance of HepG 2 cells. Consistent with previous studies reporting the resistance of HCC cells to TRAIL (10-12), human HCC HepG2 cells were relatively resistant to TRAIL-induced cytotoxicity compared with other human cancer cell lines including HCT116 colorectal cancer cells, and SNU601 and AGS gastric cancer cells. HCT116 and SNU601 cells were sensitive to as low as $2 \mathrm{ng} / \mathrm{ml}$ rhTRAIL, but HepG2 and AGS cells were less sensitive to TRAIL even at $10 \mathrm{ng} / \mathrm{ml}$ rhTRAIL (Fig. 1A). In this study, we found that HepG2 cells which show primary resistance to TRAIL become more resistant after exposure to rhTRAIL. To understand the mechanisms by which cancer cells gain stronger resistance to TRAIL, we developed stably growing cells at high concentrations of TRAIL (HepG2-TR) by exposing HepG 2 cells to stepwise increases in rhTRAIL 

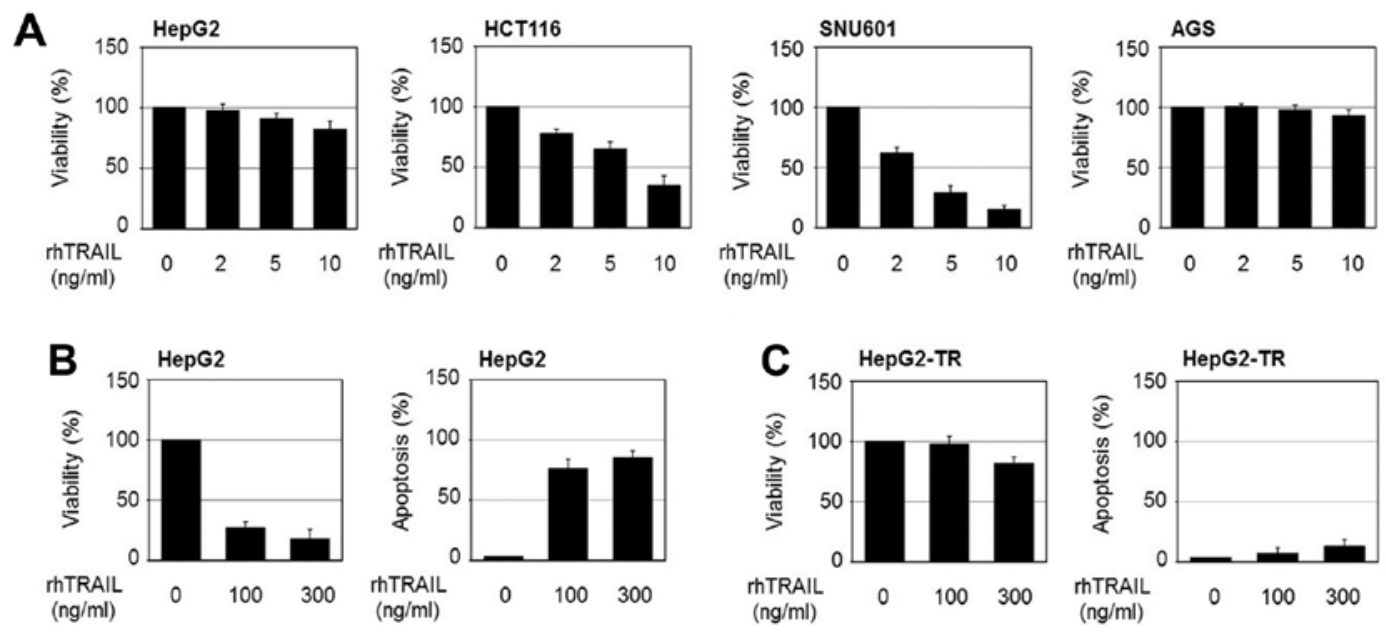

Figure 1. Sensitivity of human cancer cells to rhTRAIL. (A) Various human cancer cells, HepG2 HCC, HCT116 colon cancer cells, SNU601, and AGS gastric cancer cells were exposed to $0,2,5$ and $10 \mathrm{ng} / \mathrm{ml} \mathrm{rhTRAIL}$ for $24 \mathrm{~h}$, and cell viability was detected using an MTT assay. (B) HepG2 cells and (C) a TRAILresistant variant of HepG2 (HepG2-TR) were exposed to 0, 100 and $300 \mathrm{ng} / \mathrm{ml}$ rhTRAIL for $24 \mathrm{~h}$. Cells were analyzed using an MTT assay or Hoechst 33342 staining to measure apoptosis.
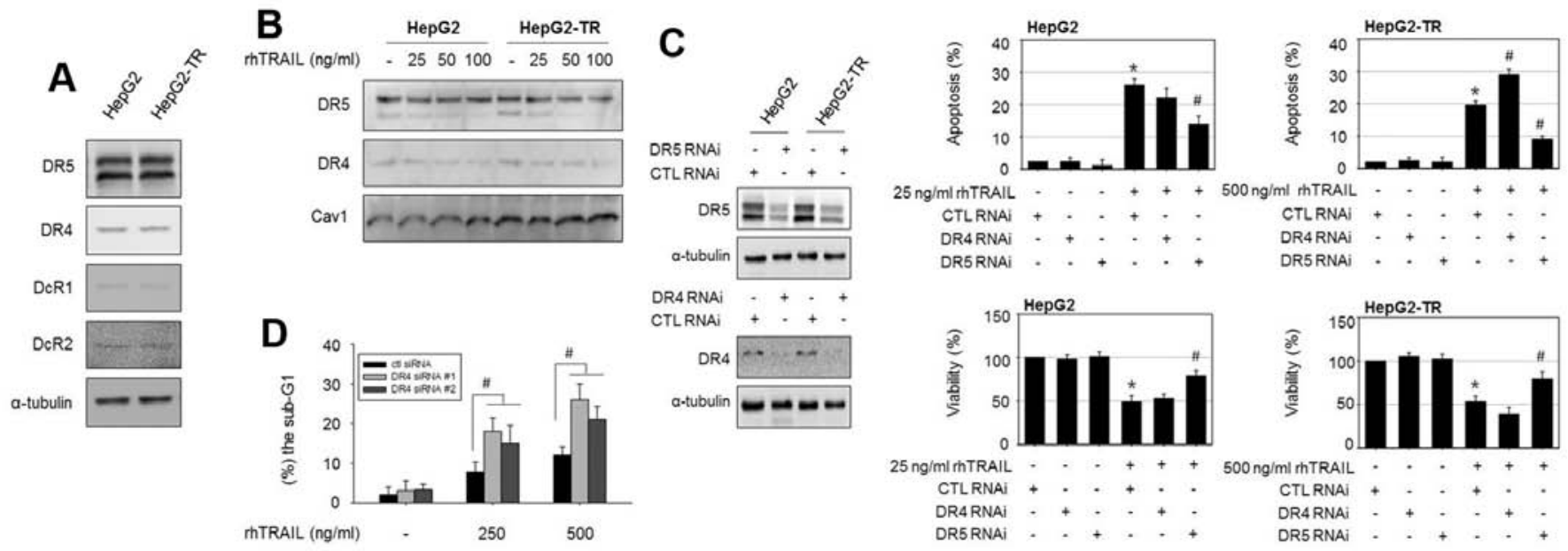

Figure 2. DR4 is involved in rhTRAIL resistance in HepG2-TR cells. (A) Total cell lysates of HepG2 and HepG2-TR cells were analyzed by immunoblotting and the expression levels of the TRAIL receptors DR4, DR5, DcR1 and DcR2 were assessed. (B) HepG2 and HepG2-TR cells were exposed to the indicated concentrations of rhTRAIL for $24 \mathrm{~h}$ and the cells were separated into membrane and non-membrane fractions. The membrane fraction was analyzed by immunoblotting. Caveolin-1 was used as a loading control. (C) HepG2 and HepG2-TR cells were transfected with DR4 siRNA, DR5 siRNA, or scrambled siRNA (CTL RNAi), and treated with vehicle or rhTRAIL ( $25 \mathrm{ng} / \mathrm{ml}$ for HepG2, $500 \mathrm{ng} / \mathrm{ml}$ for HepG2-TR) for $24 \mathrm{~h}$. They were then subjected to an apoptosis analysis (upper panels) or an MTT assay (lower panels). ${ }^{*} \mathrm{P}<0.05$ vs. control; " $\mathrm{P}<0.05$ vs. CTL RNAi transfected cells treated with rhTRAIL. (D) HepG2-TR cells were transfected with DR4 siRNA, DR5 siRNA, or control siRNA and exposed to 0,250 and $500 \mathrm{ng} / \mathrm{ml} \mathrm{rhTRAIL}$ for $24 \mathrm{~h}$. The sub-G $\mathrm{G}_{1}$ DNA content of the treated cells was quantified by flow cytometry. $\mathrm{P}<0.05$ vs. CTL RNAi transfected cells.

concentrations $(5-100 \mathrm{ng} / \mathrm{ml})$. The TRAIL-resistant cells were verified by detecting cell viability and apoptosis after rhTR AIL application. As shown in Fig. 1B and C, $<20 \%$ loss of viability was detected in the HepG2-TR cells after exposure to $300 \mathrm{ng} / \mathrm{ml}$ rhTRAIL for $24 \mathrm{~h}$, in contrast to $80 \%$ loss of viability in parental HepG2 cells. Consistently, apoptotic bodies in response to rhTRAIL were significantly decreased in the HepG2-TR cells.

DR4 is involved in the resistance to rhTRAIL-induced apoptosis in HepG2-TR cells. Alterations in the expression levels or functional activity of TRAIL receptors could be linked to the sensitivity to TRAIL in various cancer cell types. However, an immunoblotting analysis revealed that the protein levels of the death receptors DR4 and DR5 and decoy receptors DcR1 and DcR2 did not differ between HepG2-TR cells and parental HepG2 cells (Fig. 2A). Next, we analyzed the membrane expression of DR4 and DR5 in HepG2 cells and HepG2-TR cells using membranous cell fractions because the membrane localization of DRs is thought to be essential for TRAIL-induced apoptosis. However, the membrane protein levels of DR4 and DR5 were also similar between cell types (Fig. 2B). We explored whether alterations in the functional activity of death receptors are involved in the characteristic resistance of HepG2-TR cells to rhTRAIL using small interfering RNAs. DR4 and DR5 were silenced in both cells and TRAIL-induced apoptosis and viability were examined. As shown in Fig. 2C, the transfection efficiency of DR4 and DR5 

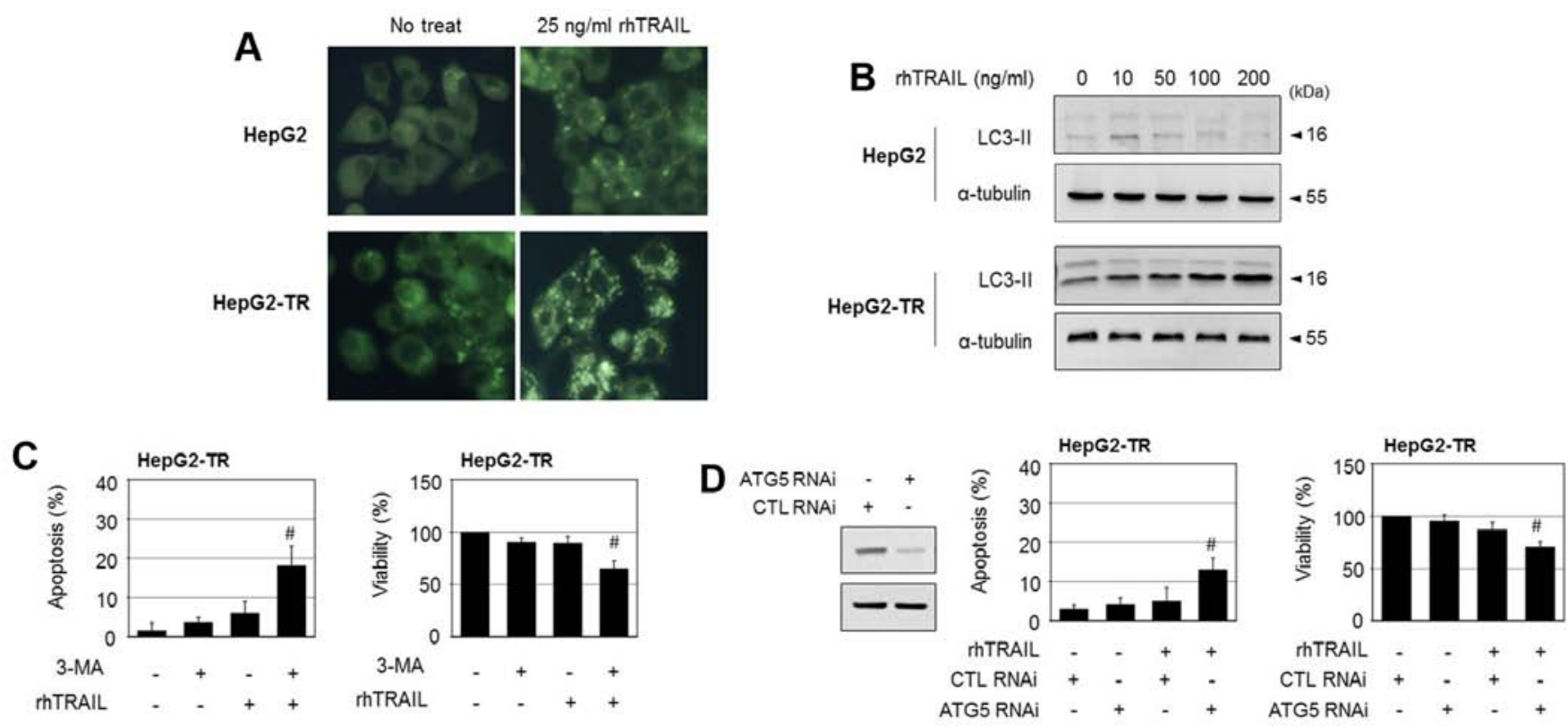

Figure 3. rhTRAIL induces protective autophagy in HepG2-TR cells. (A) HepG2 and HepG2-TR cells were treated with vehicle or $25 \mathrm{ng} / \mathrm{ml}$ rhTRAIL for $24 \mathrm{~h}$, stained with monodansylcadaverine (MDC), and observed under a fluorescence microscope (x400). (B) HepG2 and HepG2-TR cells were treated with the indicated concentrations of rhTRAIL for $24 \mathrm{~h}$ and the protein extracts were analyzed by immunoblotting against the LC3II antibody. (C and D) HepG2-TR cells were treated with vehicle or $200 \mathrm{ng} / \mathrm{ml}$ rhTRAIL for $24 \mathrm{~h}$ in the absence or presence of 3-methyladenine (C), and HepG2-TR cells transfected with ATG5 siRNA or control siRNA were exposed to $200 \mathrm{ng} / \mathrm{ml}$ rhTRAIL for $24 \mathrm{~h}$ (D). Then, the treated cells were subjected to an apoptosis analysis or an MTT assay. ${ }^{\#} \mathrm{P}<0.05$ vs. rhTRAIL (C) and ${ }^{\#} \mathrm{P}<0.05$ vs. CTL RNAi transfected cells treated with rhTRAIL (D).

siRNA was confirmed by immunoblotting. In parental HepG2 cells, $25 \mathrm{ng} / \mathrm{ml}$ rhTRAIL triggered apoptosis in $26 \%$ of cells, and silencing of both $D R 4$ and $D R 5$ reduced rhTRAILinduced apoptosis. However, in the HepG2-TR cells, the knockdown of $D R 4$ increased apoptosis, while the knockdown of DR5 attenuated TRAIL-induced apoptosis. Interestingly, we observed that DR4 silencing sensitizes HepG2-TR cells, but not parental HepG2 cells, to rhTRAIL. To further confirm the role of DR4 in rhTRAIL-induced apoptosis in HepG2-TR cells, we used additional DR4 siRNAs targeting different regions of the $D R 4$ gene and confirmed the apoptotic rate in response to rhTRAIL by a flow cytometric analysis. Similarly, transfection with DR4 siRNA (\#1 and \#2) elevated rhTRAILinduced apoptosis compared to control siRNA-transfected cells, as demonstrated by the increased proportion of cells in the sub- $\mathrm{G}_{1}$ phase (Fig. 2D). These results again suggested that DR4 interference made the HepG2-TR cells more sensitive to rhTRAIL-induced cytotoxicity.

rhTRAIL induces protective autophagy in HepG2-TR cells. A recent study suggested that TRAIL resistance is correlated with the activation of autophagy in various cancer cells. To explore whether autophagy is linked with TRAIL resistance in HepG2-TR cells, the cells were stained with monodansylcadaverine, a fluorescent compound that labels autophagic vacuoles. Upon treatment with rhTRAIL, the number of autophagic vacuoles increased in the HepG2-TR cells, but not in the parental HepG2 cells (Fig. 3A). In addition, treatment with rhTRAIL elevated the protein levels of LC3-II, a molecular indicator of autophagy, in HepG2-TR cells, but did not have a significant effect on the levels of LC3II in HepG2 cells (Fig. 3B). These results indicate that rhTRAIL triggers higher levels of autophagy in HepG2-TR cells compared to the parental cells. In response to cytotoxic stimuli, the activation of autophagy may function as a protective mechanism for survival or as a mechanism of cell death, depending on the cell type and genetic context. To determine the role of autophagy in rhTRAIL-induced cell death in HepG2-TR cells, we employed the autophagy inhibitor 3-methyladenine. While 3-methyladenine had little cytotoxicity, it increased TRAIL-induced apoptosis considerably in HepG2-TR cells, as evidenced by an increase in apoptotic nuclei, and enhanced rhTRAIL-induced cytotoxicity, as evidenced by a reduction in cell viability (Fig. 3C). To further confirm these observations, siRNA targeting ATG5 was used to prevent autophagy. The knockdown of ATG5, which was confirmed by western blotting, significantly enhanced rhTRAIL-induced apoptosis and reduced cell viability (Fig. 3D). Combined, these results suggest that autophagy triggered by rhTRAIL plays a protective role in HepG2-TR cells.

DR4 plays a role in rhTRAIL-induced protective autophagy in HepG2-TR cells. In the present study, rhTRAIL triggered protective autophagy in HepG2-TR cells, and DR4 was associated with the resistance of HepG2-TR cells to rhTRAIL. Thus, we investigated whether DR4 is related to the induction of autophagy in HepG2-TR cells. To this end, we transfected the HepG2-TR cells with DR4 siRNA, and assessed the occurrence of rhTRAIL-induced autophagy based on fluorescence staining of autophagic vesicles. As shown in Fig. 4A, the knockdown of DR4 siRNA significantly reduced the number of rhTRAIL-induced autophagic vesicles compared to that of the control RNA transfected HepG2-TR cells. Furthermore, the silencing of $D R 4$ suppressed the rhTRAIL-mediated increase in the LC3II protein level and elevated the cleavage of caspase-3 
A

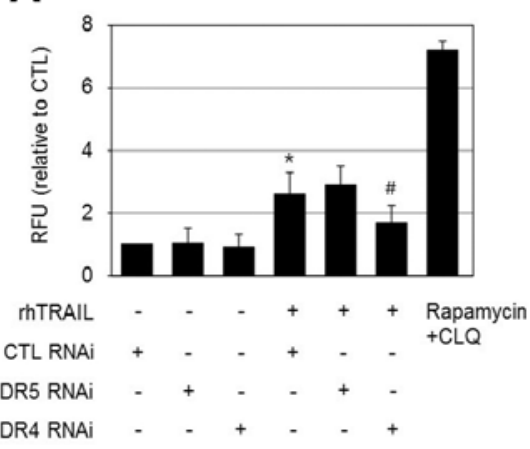

B
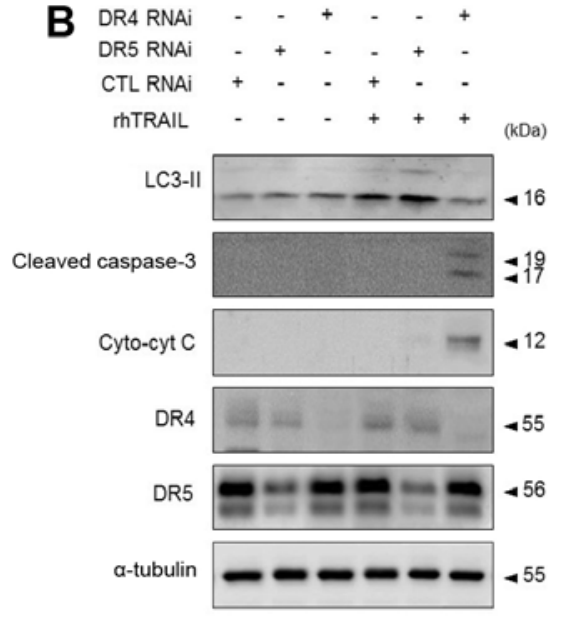

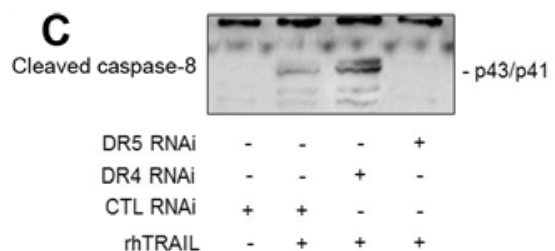

D

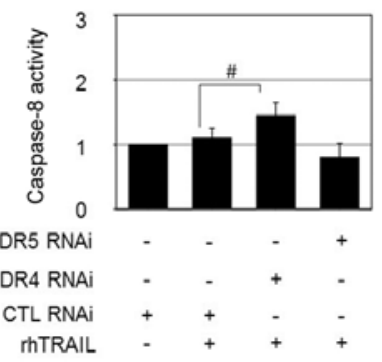

Figure 4. DR4 mediates rhTRAIL-induced autophagy in HepG2-TR cells. (A) HepG2-TR cells were transfected with DR4 siRNA, DR5 siRNA, or control siRNA, and exposed to $200 \mathrm{ng} / \mathrm{ml}$ rhTRAIL for $24 \mathrm{~h}$. Autophagy was detected using an autophagy assay kit and results are presented as relative fluorescence units. As a positive control, a combination of $1 \mu \mathrm{M}$ rapamycin and $10 \mu \mathrm{M}$ chloroquine was used. ${ }^{*} \mathrm{P}<0.05$ vs. control; " $\mathrm{P}<0.05$ vs. CTL RNAi transfected cells treated with rhTRAIL. (B-D) HepG2-TR cells transfected with DR4 siRNA, DR5 siRNA, or control siRNA were exposed to $200 \mathrm{ng} / \mathrm{ml} \mathrm{rhTRAIL}$ for $24 \mathrm{~h}$, and then subjected to immunoblotting to detect LC3II, cleaved caspase-3, cytosolic cytochrome $c$, DR4, DR5 (B), and cleaved caspase-8 (C), or to a caspase-8 activity assay (D). ${ }^{~} \mathrm{P}<0.05$ vs. CTL RNAi transfected cells treated with rhTRAIL.
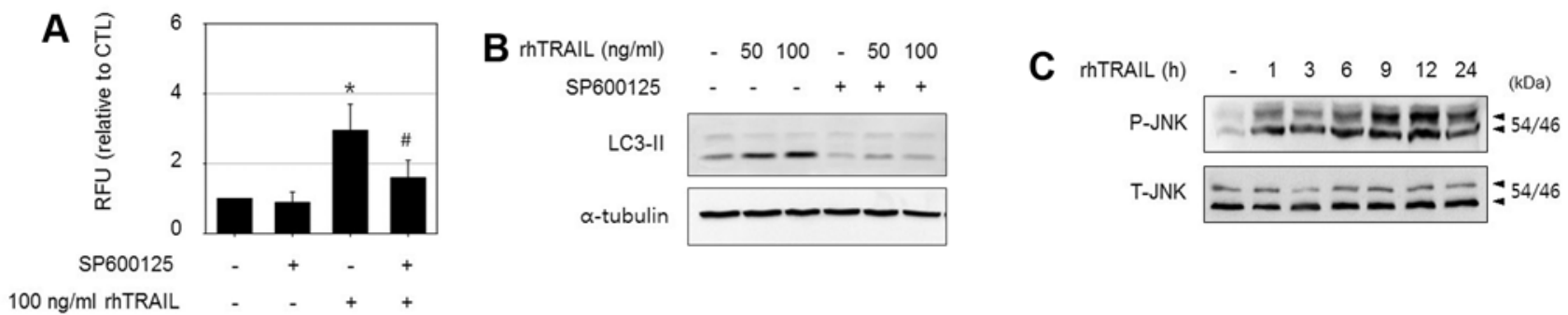

$100 \mathrm{ng} / \mathrm{ml}$ rhTRAIL $\quad-\quad+\quad+$
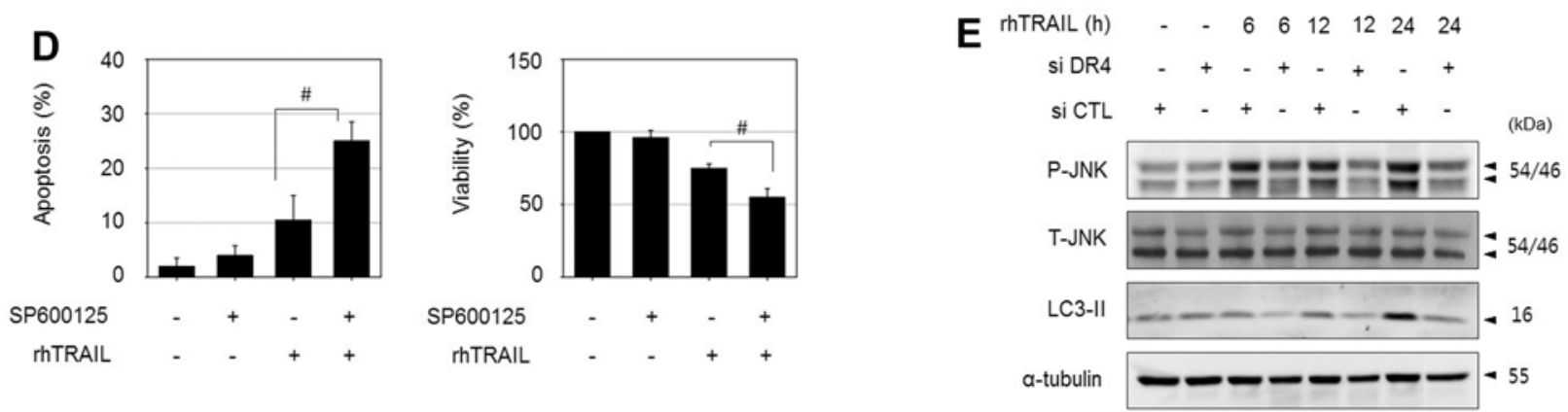

Figure 5. JNK is involved in DR4-mediated autophagy in HepG2-TR cells. (A and B) HepG2-TR cells were treated with vehicle or the indicated concentration of rhTRAIL for $24 \mathrm{~h}$ in the absence or presence of $10 \mu \mathrm{M} \mathrm{SP} 600125$. The treated cells were subjected to an autophagy assay (A), or immunoblotting to detect LC3II (B). (C) HepG2-TR cells were treated with $200 \mathrm{ng} / \mathrm{ml}$ rhTRAIL for the indicated times and analyzed by immunoblotting to detect phospho-JNK and total JNK. (D) HepG2-TR cells treated with vehicle or $200 \mathrm{ng} / \mathrm{ml}$ rhTRAIL for $24 \mathrm{~h}$ in the absence or presence of $10 \mu \mathrm{M}$ SP600125 were subjected to an apoptosis analysis or an MTT assay. (E) HepG2-TR cells transfected with DR4 siRNA or control siRNA were exposed to $100 \mathrm{ng} / \mathrm{ml} \mathrm{rhTRAIL}$ for the indicated times, and analyzed by immunoblotting to detect phospho-JNK, total JNK and LC3II. " $\mathrm{P}<0.05$ vs. control; ${ }^{\mathrm{P}} \mathrm{P}<0.05$ vs. cells treated with rhTRAIL.

and release of cytochrome $c$ in HepG2-TR cells (Fig. 4B). However, the knockdown of DR5 had no significant effect on the induction of autophagic vesicles or the elevation of LC3II levels in response to rhTRAIL. Thus, DR4 appears to play an anti-apoptotic role by contributing to the induction of autophagy in HepG2-TR cells. Furthermore, DR4 knockdown increased the levels of the active form of caspase- 8 and caspase-8 activity upon exposure to rhTRAIL in HepG2-TR cells (Fig. 4C and D). Therefore, rhTRAIL-mediated DR4 signaling may induce autophagy and interfere with DISC activation in the resistant cells.

Activation of c-Jun N-terminal kinase (JNK) is involved in DR4-mediated autophagy in HepG2-TR cells. In addition to the activation of the apoptotic pathway via DISC formation, TRAIL activates pro-survival pathways such as NF- $\mathrm{KB}$, PI3K/Akt, ERK and JNK signaling, which confer resistance to TRAIL (13-15). Furthermore, a number of studies have 
shown that the prevention of MAPK pathways can sensitize some TRAIL-resistant tumor cells to apoptosis, suggesting a possible anti-apoptotic role of MAPK $(16,17)$.

To determine the signaling molecules involved in DR4-mediated autophagy in HepG2-TR cells, we investigated the roles of several essential kinases. Among them, the JNK inhibitor SP600125 significantly reduced rhTRAIL-induced autophagy in HepG2-TR cells, as determined by an autophagy assay, and decreased the levels of LC3II (Fig. 5A and B). In addition, treatment with rhTRAIL increased phosphorylated JNK levels based on an immunoblot assay (Fig. 5C). Consistent with the cytoprotective role of autophagy, the suppression of autophagy by combined treatment with SP600125 enhanced rhTRAIL-induced apoptosis based on apoptotic body formation, and increased the sensitivity to rhTRAIL as assessed by an MTT assay (Fig. 5D). Next, we examined whether JNK activation is associated with the DR4 signaling pathway. Although inhibition of JNK using SP600125 did not affect DR4 expression level (data not shown), the silencing of DR4 reduced rhTRAIL-induced JNK phosphorylation and suppressed LC3II accumulation in HepG2-TR cells as demonstrated in Fig. 5E. These results indicate that JNK is a downstream regulator of DR4 activation. Collectively, these results suggest that the mechanism underlying TRAIL resistance is at least partially mediated by DR4/JNK-induced autophagy in HepG2-TR cells.

\section{Discussion}

Tumor cell resistance to antitumor drugs is a major obstacle in cancer therapy, including TRAIL-based approaches. Some cancer cells are intrinsically resistant to TRAIL, and even initially sensitive cancer cells often acquire stronger resistance during constant and repetitive exposure to TRAIL. The development of TRAIL resistance is attributed to the genetic heterogeneity and adaptive responses of cancer cells. These characteristics enable cancer cells to survive toxic environments and lead to the selection and expansion of survived cells after TRAIL treatment. In addition, a variety of defects that block the apoptotic cascade may be important factors in cancer cell resistance to TRAIL, and can be caused at both the receptor signaling level, e.g., diminished membrane surface expression of the TRAIL receptors DR4 or DR5 (18) and induction of c-FLIP, and at the intrinsic mitochondrial level, e.g., overexpression of the anti-apoptotic $\mathrm{Bcl}-2$ family protein MCL-1 (19). Both DR4 and DR5 are transmembrane receptors for TRAIL with cytoplasmic death domains, which can transduce apoptotic signaling via DISC formation. They share a sequence homology of $58 \%$ and induce apoptosis via similar mechanisms. However, differences in the roles of DR4 and DR5 in the induction of apoptosis have been demonstrated in various tumor cell types (20-22), and it is uncertain whether it is more advantageous to target one or both DRs for effective treatment, particularly given that the effects appear to be tumor cell-specific.

One of the essential factors that determine the differential contribution of death receptors to TRAIL-induced apoptosis may be the expression of DR4 and DR5. In agreement with this hypothesis, several antitumor agents, including ursodeoxycholic acid, andrographolide and curcumin, which increase the expression of DR4 or DR5, promote the sensitivity of various cancer cells to TRAIL $(10,23,24)$. In particular, the cell membrane expression of DRs appears to be important, since their membrane distribution is necessary for ligand binding to initiate apoptotic signaling. A decrease in the cell surface expression of DR4 or DR5 is correlated with a decrease in TRAIL sensitivity in myeloma cells and breast cancer cells $(18,25)$. Post-translational modifications, such as glycosylation and palmitoylation, of DRs are also predicted to be important mechanisms in the regulation of the sensitivity of TRAIL-induced apoptosis (26). Post-translational modification of DRs appears to be linked to the activation of death receptors, including recruitment to membrane rafts (27).

However, the TRAIL receptors DR4 and DR5 not only triggered apoptosis, but also induced survival pathways in resistant tumor cells, depending on cellular context. The known non-canonical signaling events triggered by death receptors include the activation of various kinases, such as RIP1, NF- $\mathrm{B}$, JNK, p38 kinase, ERK1/2, TAK1 and PI3K/Akt (28). In this study, we observed that DR4 is involved in TRAIL resistance in HepG2-TR cells. In parental HepG2 cells, although DR4 was much less effective than DR5 in TRAIL-induced apoptosis, DR4 did not confer resistance. Nevertheless, rhTRAIL induced autophagy in HepG2-TR cells, as detected by autophagic vacuoles and LC3II levels, and this was at least partially regulated by DR4. The knockdown of DR4 not only reduced the autophagic features, but also restored apoptosis and cytotoxicity. These results indicate that DR4 function switched to play an antagonistic role in TRAIL-induced apoptosis by triggering protective autophagy in HepG2-TR cells. Similar to our results, an anti-apoptotic role of DR4 has been reported in human lung cancer cells, in which the geranylgeranyltransferase inhibitor GGTI298 effectively augmented TRAIL-induced apoptosis in resistant cells, and in these conditions, the knockdown of DR5 attenuated apoptosis, but the knockdown of DR4 sensitized cancer cells to GGTI298/TRAIL-induced apoptosis (29).

Autophagy is an alternative mechanism for cell death, referred to as type II cell death; however, it also acts as an adaptive cell response mechanism, protecting cells from bioenergetic stress. Various cytotoxic agents that induce apoptosis activate protective autophagy in certain cancer cells $(30,31)$. TRAIL was also shown to be able to induce both apoptosis and autophagy in cancer cells. Furthermore, autophagy attenuates TRAIL-induced apoptosis and is related to the resistance of cancer cells to TRAIL treatment. The prevention of a TRAILmediated autophagic pathway by silencing autophagic genes facilitates TRAIL-induced apoptosis (32). These results are in accordance with our findings that the occurrence of autophagy in response to TRAIL confers resistance to HepG2-TR cells.

Although the mechanism by which DR4 signaling triggers autophagy is not fully understood, it appears that a downstream effector of DR4 switched to an autophagic regulator from an apoptotic DISC factor during the adaptive response to rhTRAIL, as evidenced by the suppression of caspase- 8 activation in HepG2-TR cells. In an analysis of the mechanisms involved in DR4-mediated autophagic signaling in HepG2-TR cells, we found that JNK activation is linked to the induction of autophagy. Previous studies demonstrated that TRAIL can activate the JNK pathway in several cancer cell 
lines via dual effects, cell death or survival, depending on the cellular context (33). In the present study, JNK appeared to mediate DR4-signalled autophagy, as evidenced by suppression of JNK phosphorylation after DR4 silencing and decrease in autophagy by JNK inhibition. Thus, the DR4/JNK axis may play an essential role in the TRAIL-mediated autophagic pathway in HepG2-TR cells.

However, the induction of DR4-mediated autophagy may not be the sole explanation for the resistance of HepG2-TR cells because blocking DR4 did not completely reduce cell viability. Previously, the inhibition of proteasomes by MG132 or PS-341 rescues TRAIL sensitivity in TRAIL-resistant HepG2 cells (34), suggesting that proteasomes is another resistance mechanism. It was also hypothesized that TRAILresistant HepG2 cells can be sensitized by decrease in cFLIP expression and caspase- 8 activation (11), which is consistent with our results regarding the suppression of caspase- 8 activity in resistant cells, although the resistance mechanism is different. This disparity could be explained by the different conditions with respect to TRAIL exposure; differences in the concentration and treatment duration may induce different tolerance mechanisms. Thus, even within a cell line, there appears to be various resistance mechanisms depending on micro-environmental conditions. The results of this study extend our understanding of TRAIL resistance mechanisms and may contribute to development of strategies to overcome TRAIL resistance.

\section{Acknowledgements}

The present study was supported by a research fund from the Chosun University, 2014 (K207007001). We thank Professor Tae-Hyoung Kim for the kind gift of rhTRAIL and Ms. Jeong-Eun Choi for her excellent technical assistance.

\section{References}

1. Pitti RM, Marsters SA, Ruppert S, Donahue CJ, Moore A and Ashkenazi A: Induction of apoptosis by Apo-2 ligand, a new member of the tumor necrosis factor cytokine family. J Biol Chem 271: 12687-12690, 1996.

2. Ashkenazi A, Pai RC, Fong S, Leung S, Lawrence DA, Marsters SA, Blackie C, Chang L, McMurtrey AE, Hebert A, et al: Safety and antitumor activity of recombinant soluble Apo2 ligand. J Clin Invest 104: 155-162, 1999.

3. Walczak H, Miller RE, Ariail K, Gliniak B, Griffith TS, Kubin M, Chin W, Jones J, Woodward A, Le T, et al: Tumoricidal activity of tumor necrosis factor-related apoptosis-inducing ligand in vivo. Nat Med 5: 157-163, 1999.

4. Wiley SR, Schooley K, Smolak PJ, Din WS, Huang CP, Nicholl JK, Sutherland GR, Smith TD, Rauch C, Smith CA, et al: Identification and characterization of a new member of the TNF family that induces apoptosis. Immunity 3: 673-682, 1995.

5. Falschlehner C, Emmerich $\mathrm{CH}$, Gerlach B and Walczak $\mathrm{H}$ TRAIL signalling: Decisions between life and death. Int J Biochem Cell Biol 39: 1462-1475, 2007.

6. van Dijk M, Halpin-McCormick A, Sessler T, Samali A and Szegezdi E: Resistance to TRAIL in non-transformed cells is due to multiple redundant pathways. Cell Death Dis 4: e702, 2013.

7. Rippo MR, Moretti S, Vescovi S, Tomasetti M, Orecchia S, Amici G, Catalano A and Procopio A: FLIP overexpression inhibits death receptor-induced apoptosis in malignant mesothelial cells. Oncogene 23: 7753-7760, 2004

8. Liu X, Yue P, Khuri FR and Sun SY: Decoy receptor 2 (DcR2) is a p53 target gene and regulates chemosensitivity. Cancer Res 65: 9169-9175, 2005.
9. Hetschko H, Voss V, Horn S, Seifert V, Prehn JH and Kögel D: Pharmacological inhibition of Bcl-2 family members reactivates TRAIL-induced apoptosis in malignant glioma. J Neurooncol 86: 265-272, 2008 .

10. Zhou J, Lu GD, Ong CS, Ong CN and Shen HM: Andrographolide sensitizes cancer cells to TRAIL-induced apoptosis via p53-mediated death receptor 4 up-regulation. Mol Cancer Ther 7: 2170-2180, 2008.

11. Ganten TM, Haas TL, Sykora J, Stahl H, Sprick MR, Fas SC, Krueger A, Weigand MA, Grosse-Wilde A, Stremmel W, et al: Enhanced caspase- 8 recruitment to and activation at the DISC is critical for sensitisation of human hepatocellular carcinoma cells to TRAIL-induced apoptosis by chemotherapeutic drugs. Cell Death Differ 11 (Suppl 1): S86-S96, 2004.

12. Pathil A, Armeanu S, Venturelli S, Mascagni P, Weiss TS, Gregor M, Lauer UM and Bitzer M: HDAC inhibitor treatment of hepatoma cells induces both TRAIL-independent apoptosis and restoration of sensitivity to TRAIL. Hepatology 43: 425-434, 2006.

13. Song JH, Tse MC, Bellail A, Phuphanich S, Khuri F, Kneteman NM and Hao C: Lipid rafts and nonrafts mediate tumor necrosis factor related apoptosis-inducing ligand induced apoptotic and nonapoptotic signals in non small cell lung carcinoma cells. Cancer Res 67: 6946-6955, 2007.

14. Xu J, Zhou JY, Wei WZ and Wu GS: Activation of the Akt survival pathway contributes to TRAIL resistance in cancer cells. PLoS One 5: e10226, 2010.

15. Zhang L, Dittmer MR, Blackwell K, Workman LM, Hostager B and Habelhah H: TRAIL activates JNK and NF- $\mathrm{BB}$ through RIP1-dependent and -independent pathways. Cell Signal 27: 306-314, 2015.

16. Secchiero P, Gonelli A, Carnevale E, Milani D, Pandolfi A, Zella D and Zauli G: TRAIL promotes the survival and proliferation of primary human vascular endothelial cells by activating the Akt and ERK pathways. Circulation 107: 2250-2256, 2003.

17. Zhang XD, Borrow JM, Zhang XY, Nguyen T and Hersey P: Activation of ERK $1 / 2$ protects melanoma cells from TRAILinduced apoptosis by inhibiting Smac/DIABLO release from mitochondria. Oncogene 22: 2869-2881, 2003.

18. Zhang Y and Zhang B: TRAIL resistance of breast cancer cells is associated with constitutive endocytosis of death receptors 4 and 5. Mol Cancer Res 6: 1861-1871, 2008.

19. Han J, Goldstein LA, Gastman BR and Rabinowich H: Interrelated roles for Mcl-1 and BIM in regulation of TRAIL-mediated mitochondrial apoptosis. J Biol Chem 281: 10153-10163, 2006.

20. MacFarlane M, Inoue S, Kohlhaas SL, Majid A, Harper N, Kennedy DB, Dyer MJ and Cohen GM: Chronic lymphocytic leukemic cells exhibit apoptotic signaling via TRAIL-R1. Cell Death Differ 12: 773-782, 2005.

21. Lemke J, Noack A, Adam D, Tchikov V, Bertsch U, Röder C, Schütze S, Wajant $\mathrm{H}$, Kalthoff $\mathrm{H}$ and Trauzold A: TRAIL signaling is mediated by DR4 in pancreatic tumor cells despite the expression of functional DR5. J Mol Med (Berl) 88: 729-740, 2010.

22. Kelley RF, Totpal K, Lindstrom SH, Mathieu M, Billeci K, Deforge L, Pai R, Hymowitz SG and Ashkenazi A: Receptorselective mutants of apoptosis-inducing ligand $2 /$ tumor necrosis factor-related apoptosis-inducing ligand reveal a greater contribution of death receptor (DR) 5 than DR4 to apoptosis signaling. J Biol Chem 280: 2205-2212, 2005.

23. Lim SC, Duong HQ, Choi JE, Lee TB, Kang JH, Oh SH and Han SI: Lipid raft-dependent death receptor 5 (DR5) expression and activation are critical for ursodeoxycholic acid-induced apoptosis in gastric cancer cells. Carcinogenesis 32: 723-731, 2011.

24. Jung EM, Park JW, Choi KS, Park JW, Lee HI, Lee KS and Kwon TK: Curcumin sensitizes tumor necrosis factor-related apoptosis-inducing ligand (TRAIL)-mediated apoptosis through CHOP-independent DR5 upregulation. Carcinogenesis 27: 2008-2017, 2006.

25. Gómez-Benito M, Martinez-Lorenzo MJ, Anel A, Marzo I and Naval J: Membrane expression of DR4, DR5 and caspase-8 levels, but not Mcl-1, determine sensitivity of human myeloma cells to Apo2L/TRAIL. Exp Cell Res 313: 2378-2388, 2007.

26. Gonzalvez F and Ashkenazi A: New insights into apoptosis signaling by Apo2L/TRAIL. Oncogene 29: 4752-4765, 2010. 
27. Linderoth E, Pilia G, Mahajan NP and Ferby I: Activated Cdc42-associated kinase 1 (Ack1) is required for tumor necrosis factor-related apoptosis-inducing ligand (TRAIL) receptor recruitment to lipid rafts and induction of cell death. J Biol Chem 288: 32922-32931, 2013.

28. Azijli K, Weyhenmeyer B, Peters GJ, de Jong S and Kruyt FA: Non-canonical kinase signaling by the death ligand TRAIL in cancer cells: Discord in the death receptor family. Cell Death Differ 20: 858-868, 2013

29. Chen S, Fu L, Raja SM, Yue P, Khuri FR and Sun SY: Dissecting the roles of DR4, DR5 and c-FLIP in the regulation of geranylgeranyltransferase I inhibition-mediated augmentation of TRAIL-induced apoptosis. Mol Cancer 9: 23, 2010.

30. Kondo Y, Kanzawa T, Sawaya R and Kondo S: The role of autophagy in cancer development and response to therapy. Nat Rev Cancer 5: 726-734, 2005.
31. Mizushima N, Yoshimori $\mathrm{T}$ and Levine B: Methods in mammalian autophagy research. Cell 140: 313-326, 2010.

32. Han J, Hou W, Goldstein LA, Lu C, Stolz DB, Yin XM and Rabinowich H: Involvement of protective autophagy in TRAIL resistance of apoptosis-defective tumor cells. J Biol Chem 283: 19665-19677, 2008.

33. Mahalingam D, Keane M, Pirianov G, Mehmet H, Samali A and Szegezdi E: Differential activation of JNK1 isoforms by TRAIL receptors modulate apoptosis of colon cancer cell lines. Br J Cancer 100: 1415-1424, 2009.

34. Ganten TM, Koschny R, Haas TL, Sykora J, Li-Weber M, Herzer K and Walczak H: Proteasome inhibition sensitizes hepatocellular carcinoma cells, but not human hepatocytes, to TRAIL. Hepatology 42: 588-597, 2005. 